\title{
Are the sungrazing comets the inner source of pickup ions and energetic neutral atoms?
}

\author{
M. Bzowski and M. Królikowska
}

\author{
Space Research Centre PAS, Bartycka 18A, Warsaw, Poland \\ e-mail: bzowski@cbk.waw.pl
}

Received 27 April 2004 / Accepted 31 January 2005

\begin{abstract}
We present arguments that at least part of the inner source of pickup ions in the solar wind might be the material released by sungrazing comets. Based on a statistical analysis of sungrazing comets detected over almost eight years of LASCO operation (1996 - September 9, 2004) an overwhelming majority of the observed sungrazers belong to the Kreutz group of comets, follows tightly clumped orbits and break up at $\sim 40-4$ solar radii in a well defined region of space. The material released from these comets could be (after ionization) an important portion of the inner source of pickup ions (PUIs), as the local mass flux of the inner source and cometary PUIs seem comparable. We indicate time intervals during the year when the cometary PUIs could be observed from a spacecraft on the Earth's orbit (from the end of July until the end of the year) and show three time intervals when they should be observable by Ulysses (from its launch time until the end of 1990, from the end of November 1994 until mid-May 1995 and from February 2001 until the end of July, 2001). We argue that the PUIs from the inner source should include both singly and doubly charged ions and that this cometary hypothesis alleviates some difficulties (in particular, the issue of hydrogen deficit) in the interpretation of the inner source as solar wind neutralized on dust grains close to the Sun.
\end{abstract}

Key words. solar wind - comets: general - interplanetary medium

\section{Introduction}

Pickup ions (PUI) in the solar wind are former neutral atoms of thermal energy, ionized and picked up by the solar wind (Fahr 1973). $\mathrm{He}^{+}$PUI created from interstellar atoms penetrating the heliosphere were first detected by Möbius et al. (1985) and $\mathrm{H}^{+}$by Gloeckler et al. (1993). The inner source of pickup ions was discovered by Geiss et al. (1994, 1995). Its elemental composition, which includes oxygen (mass 16), nitrogen (mass 14), neon (mass 20), hydrogen, carbon (mass 12), magnesium (mass 24) and silicon (mass 28), identified by the mass/charge ratio (Gloeckler \& Geiss 1998; Gloeckler et al. 2000a), is quite similar to the solar wind composition. This suggests that the inner source is related to dust or that this portion of the PUI population must be solar wind ions neutralized, slowed down and reionized. Analysis of the inner source PUI velocity distribution function indicates that the source must be located very close to the Sun $(<0.1 \mathrm{AU})$.

In this communication we suggest that at least part of the inner source of pickup ions might be the material released by sungrazing comets.

Shortly after beginning of operations, the LASCO coronograph on-board the SOHO spacecraft discovered a stream of comets approaching the Sun to a few solar radii (Biesecker et al. 2002). Until early September 2004, almost 850 such objects have been observed and orbits of 809 have been determined.
We present statistical analysis of these 809 SOHO sungrazers based on the orbital data taken from the Catalogue of Cometary Orbits (Marsden \& Williams 2003) and from IAU Circulars publicly available on the Web at http://cfa-www.harvard. edu/iauc/RecentIAUCS.html (Cambridge, USA). We confirm the earlier finding by Biesecker et al. (2002) that the sungrazers are in fact more frequent than observed and we postulate that due to this high frequency of apparitions they should provide a source of pickup ions which is virtually ever-present and wellconstrained in space. We indicate time intervals during the year when the sungrazer comet-related PUI should be observable by Earth- (and L1)- bound spacecraft and the portions of the Ulysses orbit where these ions should reach the spacecraft. We discuss Energetic Neutral Atoms (ENA) created due to charge exchange between solar wind protons and cometary neutral atoms and we conclude that owing to reionization in the proximity of the Sun they should create an extra source of "inner source" pickup protons.

\section{Statistics of orbits and perihelion time of sungrazing comets}

An overwhelming portion (86\%) of the sungrazing comets belongs to the Kreutz group. The Kreutz group of comets was discovered in the 19th century (Kreutz 1888, 1891, 1901) 


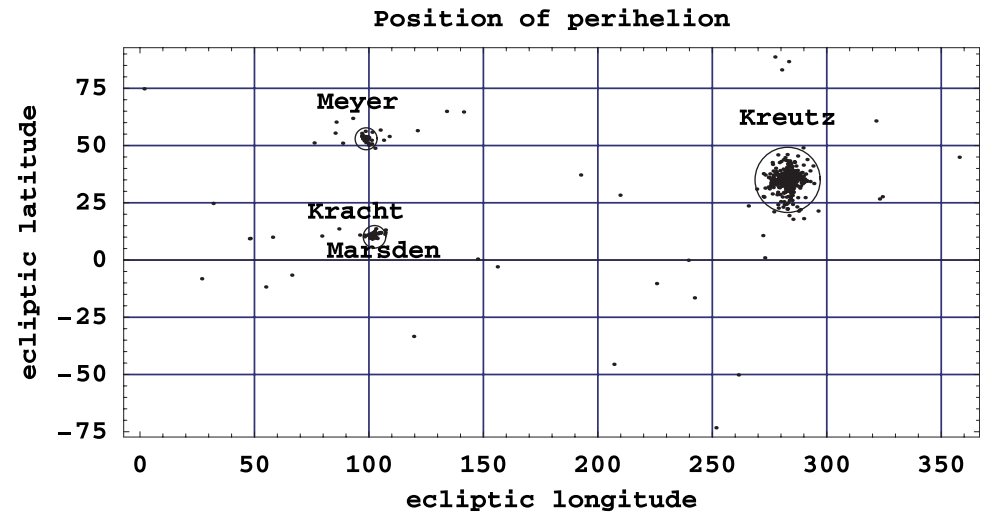

Fig. 1. Distribution of ecliptic coordinates of perihelion points of known SOHO sungrazers. For October 2004, the primary Kreutz group has 686 members, the Meyer group 40 and the Marsden \& Kracht group together 39. Circles mark the angular distances around respective mean values containing $99 \%$ of the members of the three groups.
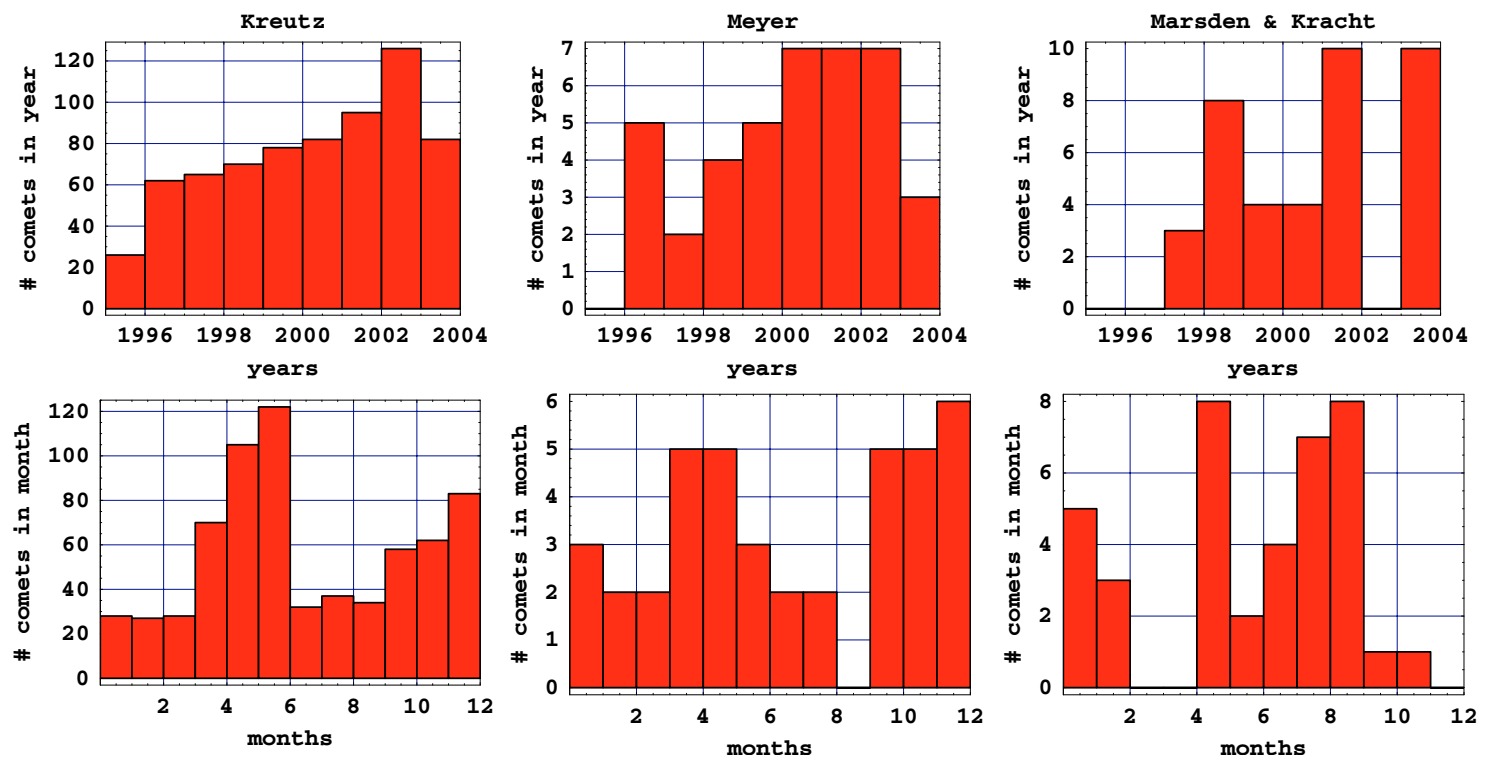

Fig. 2. Histograms of perihelion passage times for the SOHO sungrazing groups of comets: upper panel - yearly, lower panel - monthly. The pattern for the monthly rates of the Kreutz group holds for each year within statistical uncertainty.

and observed also later throughout the 20th century: visually (Marsden 1967) and by space-borne SOLWIND, SMM (Sheeley et al. 1982; Michels et al. 1982; Marsden 1989; MacQueen \& StCyr 1991), and LASCO coronographs. The pre-LASCO observations discovered 46 sungrazing comets. After the initial period of LASCO observations, the discovery rate quickly increased and since 1997 it has been oscillating between 60 and 120 comets per year with orbital elements determined (Fig. 2, upper-left panel; see also Biesecker et al. 2002). Hence we believe that the Kreutz group has been a persistent phenomenon at least during the era of in situ space exploration, presumably with a weakly changing annual rate.

In addition to the Kreutz group, there are three additional groups of sungrazing comets, by far less numerous than the Kreutz group but nevertheless non-negligible as a potential source of the "inner source" PUIs: Meyer (5\% of all SOHO sungrazers), Marsden (2\%), and Kracht (3\%). Their orbital parameters seem correlated in a certain way with the Kreutz group. These four groups include almost all known sungrazing comets (except 35 "stragglers").

Comets from the Kreutz group follow extremely elongated elliptical orbits (Sekanina 2002a). Because of poor quality of
Table 1. Mean and most probable orbital parameters of the Kreutz group of sungrazing comets; total of 686 comets in the sample.

\begin{tabular}{lcccc}
\hline \hline Parameter & Value & $\begin{array}{c}\text { Std. } \\
\text { Dev. }\end{array}$ & $\begin{array}{c}\text { Most } \\
\text { probable }\end{array}$ & $\begin{array}{c}\text { Mean from } \\
\text { Sekanina }\end{array}$ \\
\hline Perihelion longitude $\left[{ }^{\circ}\right]$ & 282.9 & 2.9 & 283. & 282.6 \\
Perihelion latitude $\left[{ }^{\circ}\right]$ & 35.0 & 3.4 & 35. & 35.2 \\
Perihelion distance $\left[R_{\odot}\right]$ & 1.22 & 0.22 & 1.07 & 1.10 \\
Inclination $\left[{ }^{\circ}\right]$ & 143.5 & 2.8 & 144. & 144.5 \\
Perihelion argument $\left[{ }^{\circ}\right]$ & 80.1 & 12.0 & 85. & 83. \\
Node $\left[{ }^{\circ}\right]$ & 1.0 & 14.4 & 7. & 4. \\
\hline
\end{tabular}

positional data, all orbits of SOHO sungrazers could only be determined with the assumption that their eccentricity is equal to 1 . The ecliptic coordinates of perihelia of all LASCO sungrazers with known orbits, detected before September 9, 2004, are shown in Fig. 1 and their mean orbital elements in Tables 1 and 2. The Marsden and Kracht groups, although slightly different to each other, have such similar trajectories close to the Sun that in this paper we will treat them together as the 
Table 2. Mean orbital parameters of the Meyer and Marsden \& Kracht groups of sungrazing comets (respectively, a total of 40 and 39 comets in the samples).

\begin{tabular}{lcc}
\hline \multicolumn{3}{c}{ Meyer Group } \\
Parameter & Value & Std. dev. \\
\hline Perihelion longitude $\left[{ }^{\circ}\right]$ & 98.8 & 1.3 \\
Perihelion latitude $\left[{ }^{\circ}\right]$ & 53.0 & 1.4 \\
Perihelion distance $\left[R_{\odot}\right]$ & 7.7 & 0.4 \\
Inclination $\left[{ }^{\circ}\right]$ & 72.2 & 1.3 \\
Perihelion argument $\left[{ }^{\circ}\right]$ & 57.0 & 1.2 \\
Node $\left[{ }^{\circ}\right]$ & 73.6 & 1.3 \\
\hline \multicolumn{1}{c}{ Marsden $\mathcal{E}$ Kracht Group } \\
Parameter & Value & Std. dev. \\
\hline Perihelion longitude $\left[{ }^{\circ}\right]$ & 101.9 & 2.4 \\
Perihelion latitude $\left[{ }^{\circ}\right]$ & 10.8 & 1.1 \\
Perihelion distance $\left[R_{\odot}\right]$ & 9.90 & 0.96 \\
Inclination $\left[{ }^{\circ}\right]$ & 18.5 & 6.9 \\
Perihelion argument $\left[{ }^{\circ}\right]$ & 45.5 & 19.0 \\
Node $\left[{ }^{\circ}\right]$ & 57.7 & 20.0 \\
\hline
\end{tabular}

Marsden \& Kracht group. Since there were no reports on apparitions of comets from these groups before LASCO, it may be - especially in the case of the Marsden \& Kracht group that they are transient phenomenon lasting just a few years. On the other hand, even the Kreutz comets were observed rarely and far in between before LASCO, so it cannot be ruled out that the lack of earlier apparitions is a selection effect.

The yearly rate of sungrazing comets from all groups is most probably biased by selection effects related to the construction of the LASCO coronographs (Biesecker et al. 2002). When binned monthly, all groups show a statistically significant seasonal variability of the apparition rate, featuring one sharp peak and a broader secondary peak 6 months later (Fig. 2, lower panel). For the Kreutz group, the monthly pattern shown in Fig. 2 persists for each separate year (within deviations due to the duty cycle of the LASCO instrument and statistical spread); the other groups are too scarce for a statistically significant analysis of this phenomenon on a yearly basis. The primary peak of the Kreutz group occurs in June and the secondary in December (see Fig. 2, lower-left panel). The monthly peaks of the Meyer group correspond well with the Kreutz peaks (Fig. 2, second panel in the lower row), while the peaks of the Marsden \& Kracht group seem to be shifted in time by about 2 months (Fig. 2, lower right panel).

The highest monthly rate during the whole LASCO observation period occurs in June and we adopt this rate as the true rate of the Kreutz group. During the seven full years of LASCO observations (1997-2003) about 100 comets were observed during this month. Thus we conclude that the true apparition rate is 100:7 $\simeq 14$ per month, or one every other day. Based on a much smaller sample, observed from the end of 1996 until the end of 1998, Sekanina (2003) gives a similar estimate for the apparition rate: 0.6 per day. The peak monthly rates for the Meyer and Marsden \& Kracht groups are, correspondingly, 5 and 8 during the $\sim 7$ years period, which yields about one per month in each of the two groups.

Based on the lightcurves of $\sim 20$ brightest objects, Sekanina (2003) gives an estimate of the number $N_{\text {cum }}$ of comets with the mass greater than or equal to $M$ as:

$N_{\text {cum }}(M)=\left(\frac{M_{0}}{M}\right)^{v}=22\left(\frac{10^{10} \mathrm{~g}}{M}\right)^{0.68}$

(with the selection effects taken into account) and of the total mass $M_{\text {sum }}$ carried by the train of Kreutz comets with masses lower than or equal to $M$ as:

$M_{\text {sum }}(M)=\frac{v}{1-v} M_{0}\left(\frac{M_{0}}{M}\right)^{v-1}$.

In these equations, $M_{0}$ is an unknown mass of the most massive comet observed and $v=0.68$ is a population index fitted by Sekanina based on the lightcurves observed. We find the mass of the most massive comet $M_{0}$ from Eq. (1) by substituting $N_{\text {cum }}=1$ and we obtain $M_{0}=0.94 \times 10^{12} \mathrm{~g}$. The total mass of the incoming comets is thus computed as $M_{\text {sum }}\left(M_{0}\right)$ and given by the formula:

$M_{\text {sum }}=M_{0} v /(1-v)$.

This gives $M_{\text {sum }}=2 \times 10^{12} \mathrm{~g}$ arrived during the $\sim 2$ years long interval, equivalent to $3.1 \times 10^{4} \mathrm{~g} \mathrm{~s}^{-1}$.

Modelling by Iseli et al. (2002) returns the most massive element at $0.63 \times 10^{12} \mathrm{~g}$, which agrees with the observational value to $\sim 30 \%$. However, observations of several bright sungrazers by Raymond et al. (1998) and Uzzo et al. (2001) suggest that their diameters are 5-fold smaller than inferred by Sekanina and Iseli, which indicates a substantially smaller mass. Since the width of the Ly- $\alpha$ line observed by UVCS suggests that those actually seen are not the original H-atoms from the comet but solar wind protons chargeexchanged with cometary atoms, we adopt results of the direct estimates, $3.1 \times 10^{4} \mathrm{~g} \mathrm{~s}^{-1}$, by Sekanina (2003).

Typical sungrazing comets disintegrate between 40 and $4 R_{\odot}(0.19-0.019 \mathrm{AU})$, typically at $10 R_{\odot} \simeq 0.05 \mathrm{AU}$ (Sekanina 2003). Very few of the comets (if any) make it through perihelion. After breakup, almost the whole mass is transferred to the solar wind as pickup ions. Additionally, the charge exchange ionization with solar wind protons produces Energetic Neutral H Atoms (H ENA) at energies from $\sim 30 \mathrm{eV}$ to $\sim 0.8 \mathrm{keV}$.

\section{Opportunities for pickup ion and ENA observations}

\subsection{Pickup ions}

\subsubsection{Seed population}

The material released from comets during the approach phase includes water molecules and $\mathrm{CO}_{2}$ and $\mathrm{CO}$ at the abundance of $3-10 \%$ and $0.5-20 \%$ with respect to water. Other molecules and atoms are much less abundant, below $1 \%$ by number 
(Geiss \& Altweg 1998; Boclée-Morvan et al. 2004). Hence the cometary PUIs from the approach phase should be almost entirely hydrogen and oxygen ions and the number abundance of oxygen with respect to hydrogen prior to ionization should be roughly 0.5 .

The mass composition of cometary nuclei given by Greenberg \& Li (1999) involves $\sim 26 \%$ of water, $\sim 23 \%$ of organic refractory material (dominated by carbon), $\sim 9 \%$ of carbon itself and $\sim 26 \%$ of silicates, whose chemical composition can be approximated by $[\mathrm{Mg}, \mathrm{Fe}]_{2} \mathrm{SiO}_{4}$. $\mathrm{CO}, \mathrm{CO}_{2}, \mathrm{CH}_{3} \mathrm{OH}$ and $\mathrm{H}_{2} \mathrm{CO}$ make up $\sim 11 \%$ the of nucleus by mass, and all other add up to $\sim 5 \%$. Based on these data we estimate that the nucleus is composed of $\sim 43 \% \mathrm{H}$ atoms, $\sim 27 \% \mathrm{O}$ atoms and $\sim 25 \% \mathrm{C}$ atoms; magnesium, silicon, iron and other atoms are only $\sim 5 \%$ of the total number density.

The dissociation rates of the cometary molecules are usually much higher than the ionization rates of ions, so one expects that all the material injected into the solar wind in the breakup region (including also the dust grains, Mann et al. 2004) will be totally dissolved into atomic ions. Considering this, and the atomic weights, one obtains the atomic production rate equal to $\sim 2 \times 10^{27} \mathrm{~s}^{-1}$. Iseli et al. (2002) estimate that oxygen and carbon from the breakup region when observed at 1 AU should be in high ionization states because of EUV secondary ionization after pickup. On the other hand, in the case of material injected into the solar wind during the approach phase, i.e., at larger heliocentric distances, one can expect to observe some molecules (mainly water and hydrocarbon dissociation products) and the probability of survival of PUIs in the singly charged state is higher.

The composition of the material released during the breakup should differ from the composition of volatiles released during the approach phase. The cometary material also involves dust; in fact most of the heavy elements in cometary nuclei are locked in dust grains, which are released from the nucleus into the surrounding space but continue towards the Sun. Some researchers hypothesize that sungrazing comets may be the main source of dust very close to the Sun, inside $\sim 3 R_{\odot}$ (Mann et al. 2004). Those dust grains will interact with the solar wind and solar radiation and additional atoms and molecules will be inserted into the solar wind. "Fresh" cometary dust, especially released from inside the breaking nucleus at a few solar radii should sublimate quickly to become dissociated/ionized and picked up by the solar wind. Kimura et al. (2002) suggest that the dust observed in the tails of sungrazing comets consists mainly of crystalline silicates and perhaps also of amorphous pyroxene at distances farther than $20 R_{\odot}$. This is consistent with the suggestion by Greenberg \& $\mathrm{Li}$ (1999) that cometary nuclei contain dust grains of a silicate core and organic refractory mantle with external mantles of water ice with carbonaceous and PAH particles embedded; very close to the Sun the mantles sublimate almost immediately after release of the grain from the nucleus, and the "rocky" silicate cores sublimate between 4 and $1.5 R_{\odot}$ (Mann et al. 2004). This is about the distance of perihelia of the sungrazing comets. Hence we conclude that even the most resistant dust cores will not survive the perihelion passage and the entire cometary material will be evacuated with the solar wind as pickup ions.

\subsubsection{Ionization and pickup}

The molecules released from the nucleus with a relative velocity of $\sim 1 \mathrm{~km} \mathrm{~s}^{-1}$ continue on the original orbits until they become dissociated by solar radiation and then ionized by photoionization, electron impact or charge exchange with the solar wind. Once created, the new ions from the cometary material are immediately picked up by solar wind and transported away from the Sun.

Because orbits of most of the sungrazing comets are tightly clumped in space, the region where the PUIs are created is well defined. Comets are known to release gas and dust at $\sim 5 \mathrm{AU}$, the aphelion of the Ulysses orbit. Thus the source region for sungrazer PUIs will be the volume of space traversed by the Kreutz comets, extended by the typical size of cometary neutral gas clouds, which is of the order of the Sun's diameter (Mäkinen et al. 2001a,b; Povich et al. 2003).

The dissociation rate of water into $\mathrm{OH}$ and $\mathrm{H}$ at 1 AU by solar radiation (the dominant ionization channel) is $\sim 13 \times 10^{-6} \mathrm{~s}^{-1}$ (Budzien et al. 1994) and the order of magnitude of a typical ionization/dissociation rate of cometary molecules and atoms at $1 \mathrm{AU}$ is $10^{-7}-10^{-5} \mathrm{~s}^{-1}$ (Huebner et al. 1992) which gives a lifetime of the order of days to months. Assuming the lifetimes decrease as $r^{-2}$, we have lifetimes from 1 to $100 \mathrm{~h}$ at $40 R_{\odot}$ and from 0.01 to $1 \mathrm{~h}$ at $4 R_{\odot}$. Comparing the travel time from $40 R_{\odot}$ to $4 R_{\odot}(\sim 50 \mathrm{~h})$ with the apparition rate of new sungrazing comets (about three comets per week, i.e., one every $50 \mathrm{~h}$ ) we conclude that almost permanent although highly fluctuating production of pickup ions could be observed. Since the final breaking occurs no closer than $4 R_{\odot}$ and the ionization times at $4 R_{\odot}$ are less than $1 \mathrm{~h}$, we estimate that the seed material can approach not closer than $2 R_{\odot}$ from the Sun's center. According to Mann et al. (2004) all dust grains must evaporate before reaching 1.4-2 $R_{\odot}$. Thus, we conclude that the source region for PUI from sungrazers extends to $\sim 2 R_{\odot}$.

\subsubsection{PUI propagation and detection}

Pickup ions from solar system bodies were observed at surprisingly large distances of millions of kilometers: Grünwaldt et al. (1997) discovered the Venus PUI tail from the Earth's orbit, Gloeckler et al. (2000b) detected the PUI tail from Comet C/1996 B2 Hyakutake, and Gloeckler et al. (2004) observed an oxygen and carbon PUI signal that shows characteristics resembling the sungrazer signature discussed by Iseli et al. (2002).

To determine the prospective region for detection of sungrazer PUIs, we make the important assumption that PUIs in the solar wind propagate radially away from the Sun whatever their injection velocity and heliocentric distance of their injection point.

Trajectories of singular $\mathrm{O}^{+}$PUIs in the solar wind (scatterfree approximation) were calculated by Luhmann (2003). Among other cases, she considered a parent oxygen atom, which at $0.3 \mathrm{AU}$ from the Sun has a velocity of $70 \mathrm{~km} \mathrm{~s}^{-1}$ and becomes ionized. Such parameters correspond almost exactly to the trajectories of the sungrazing comets at this heliocentric distance. Luhmann showed that when the pitch angle is not 


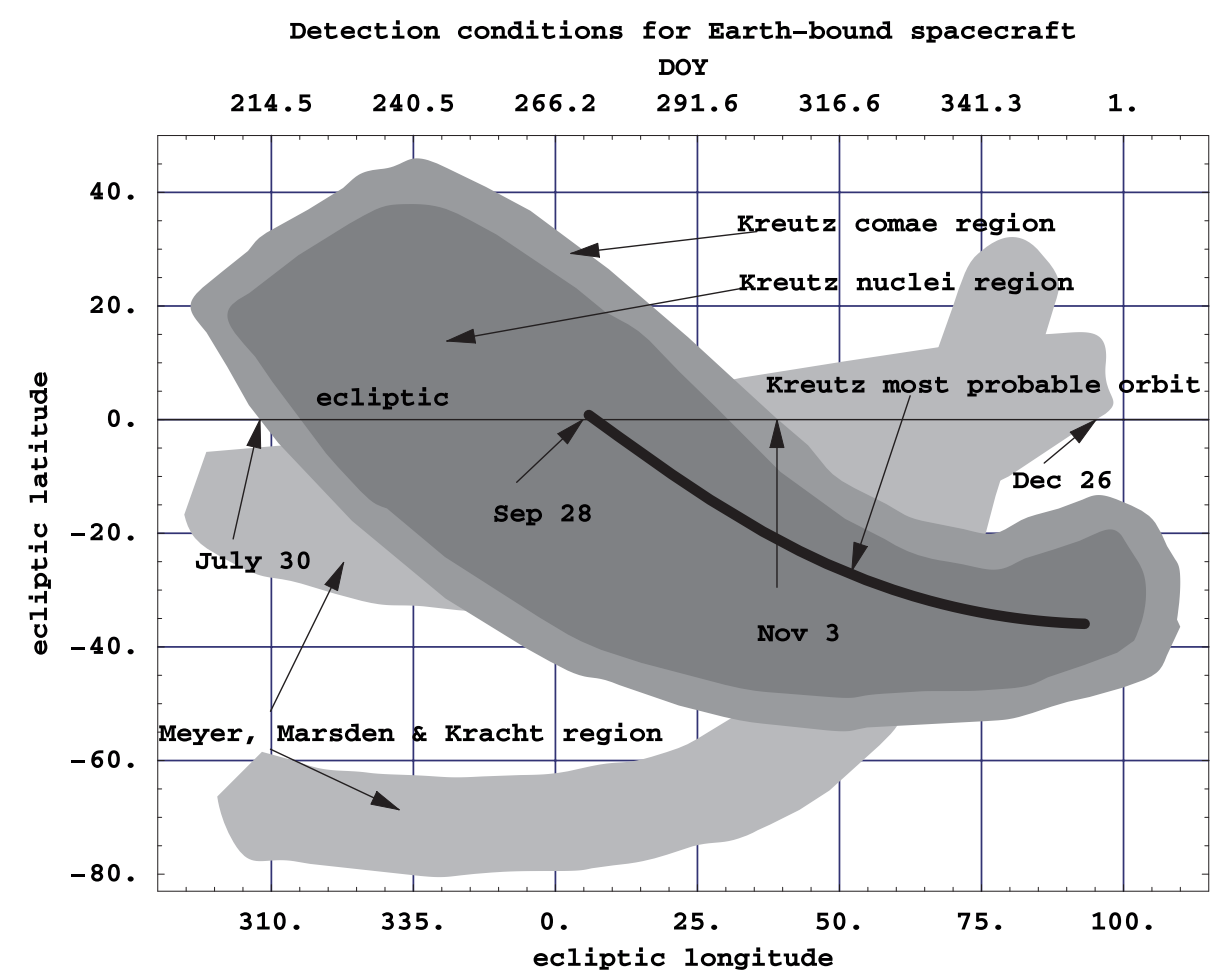

Fig. 3. Detection chart for sungrazing comet-related PUI and ENA by Earth-bound spacecraft. The dark region is a projection on sky of the volume penetrated by nuclei of the Kreutz group comets. Its lighter envelope is a projection of the volume filled by their comae. The light gray streaks are sky projections of volumes penetrated by Meyer and Marsden \& Kracht comets (with the comae). The black line is a sky projection of the most probable orbit of the Kreutz group. Arrows mark the dates of Earth entering and exiting the region prone for detection of the sungrazing comets populations and the date of the intersection with the projection of the most probable Kreutz orbit. The lower horizontal axis is scaled in ecliptic longitude and the upper axis in the corresponding DOY values; the vertical axis is ecliptic latitude.

scattered, the assumption of radial propagation of PUIs from their injection point is reasonable only for injection distances of a few $\mathrm{AU}$ from the Sun. Inside $1 \mathrm{AU}$ the propagation is not radial. When starting at $0.3 \mathrm{AU}$ and $70 \mathrm{~km} \mathrm{~s}^{-1}$, the PUIs propagate almost perpendicularly to the Sun - injection point line, and at $1 \mathrm{AU}$ this angle is $\sim 45^{\circ}$.

On the other hand, it is highly improbable that there is no scattering of the pitch angle. Pitch angle scattering would tend to make the trajectories better aligned with the Sun - injection site line. Also, one cannot neglect the influence of local modifications (draping) of the interplanetary magnetic field by the comet and their consequences for cometary PUI motion (Luhmann et al. 1988). Gloeckler et al. (1986) observed almost radial propagation of PUIs from Comet 21P/Giacobini-Zinner from a few million kilometers in what seemed like a magnetic tail and Gloeckler et al. (2000b) saw a close to radial propagation of PUIs from Comet C/1996 B2 Hyakutake. Admittedly, much farther-from-radial propagation is reported by Gloeckler et al. (2004), but in this case the parent body of the observed signal has not been unambiguously identified and there is a possibility that the PUIs had been deflected by a CME. All this makes us adopt the simplest assumption of radial propagation of PUIs (with a few degrees of broadening) both from the tails and from the breaking up nuclei of the sungrazing comets.

To find the range of ecliptic coordinates subtended by the sungrazing comets and their comae, we computed actual trajectories on the inbound (pre-perihelion) leg of all known LASCO comets from the Kreutz, Meyer and Marsden \& Kracht groups between $1 \mathrm{AU}$ and the breakup region at a few solar radii from the Sun and plotted the envelope (see Figs. 3 and 4). For all comets, we assumed an eccentricity equal to 1 (parabolic orbit), which - given eccentricities $\sim 0.999$ of those few comets for which it was possible to determine - is a sufficient approximation inside $1 \mathrm{AU}$. Additionally, for the Kreutz group we selected the most probable orbital elements, taking them as peak values from the histograms of the orbital parameter distribution. A track of this orbit is superimposed on the plots in Figs. 3 and 4 and is supposed to be the region of the highest flux of the PUIs and ENAs resulting from the cometary material. We list the parameters of this orbit along with the mean orbital elements from our sample in Tables 1 and 2 we supplement them with the mean values of orbital parameters of the Meyer and Marsden \& Kracht groups. We construct our charts of detection regions of sungrazer PUIs as a radial projection of the source regions on sky. For the Kreutz group, we show separately the region corresponding to the volume traversed by the comets themselves and the envelope, assumed to be $5^{\circ}$ wide, due to their neutral-gas clouds.

Earth- and an L1-orbiting spacecraft travel at ecliptic latitude 0 and enter the region of PUI flux from the Kreutz comets each year on about July 30 (DOY 211) and exit it on November 3 (DOY 307). For the following 2 months they are still inside the region of PUIs from the secondary groups, exiting it on December 26 (DOY 360). During that time, we expect 


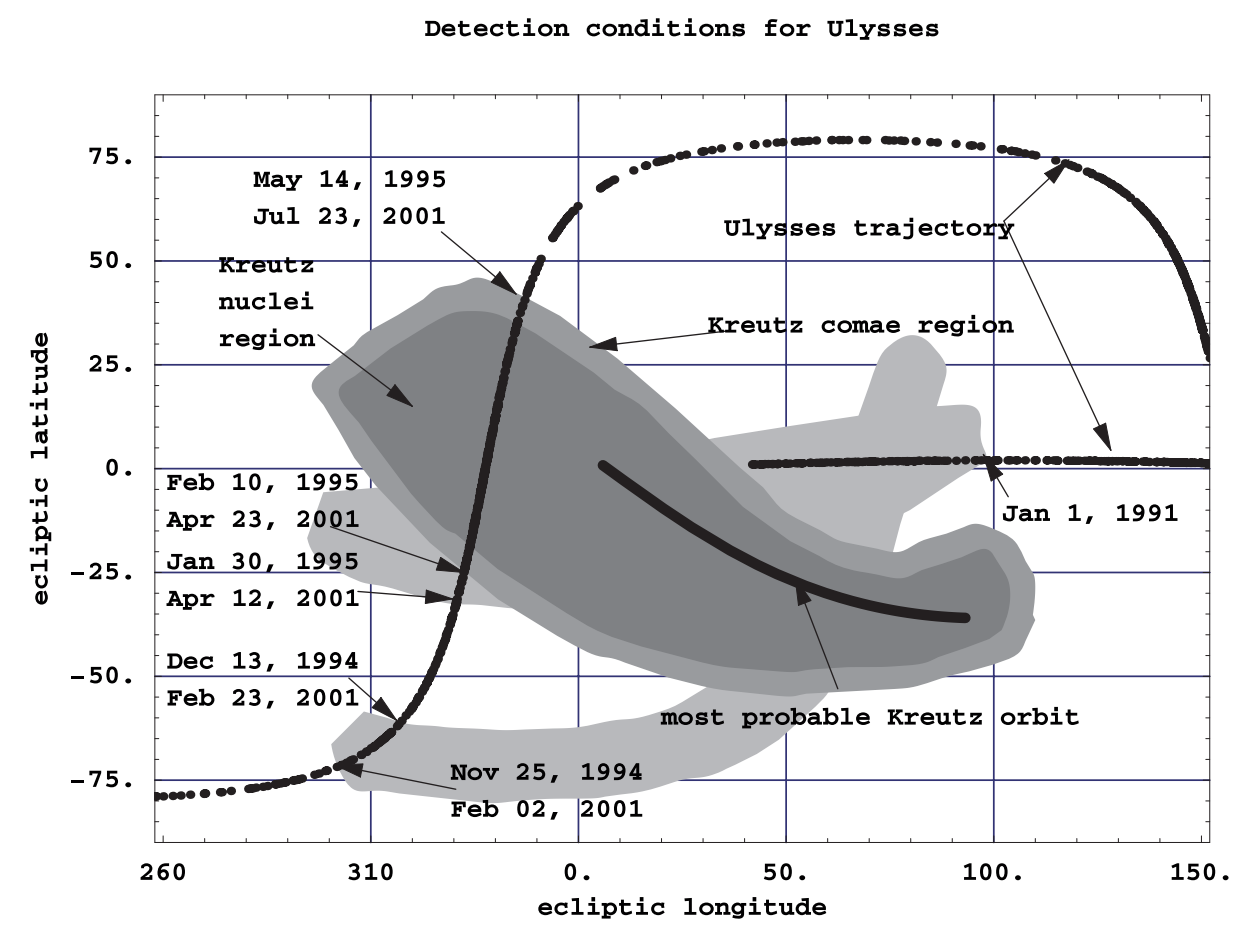

Fig. 4. Detection chart for the sungrazing comet-related PUI by Ulysses. The gray regions are identical as in Fig. 3. The Ulysses trajectory is marked with the dotted line. Ulysses was launched while in the Meyer, Marsden \& Kracht region and exited it on January 1, 1991. Then, on its two out-of-ecliptic revolutions around the Sun, it crossed the regions prone to detection of the sungrazer population on the days indicated in the figure.

Table 3. Detection intervals for sungrazing comet-related PUI and ENA for Earth-bound spacecraft ${ }^{a}$.

\begin{tabular}{lcccc}
\hline \hline Comet group & Kreutz & Kreutz & Kreutz & Meyer, Marsden \& Kracht \\
event & in & maximum & out & out \\
\hline Date & July 30 & Sep. 28 & Nov. 3 & Dec. 26 \\
DOY & 211 & 271 & 307 & 360 \\
Ecliptic longitude $\left[{ }^{\circ}\right]$ & 306 & 5 & 39 & 95 \\
\hline
\end{tabular}

${ }^{a}$ See also Fig. 3.

48 comets each year from the Kreutz group and 4 from the secondary groups. The passage through the sky projection of the orbit of the most probable Kreutz comets occurs on September 28 (DOY 271). These dates and longitude intervals are given in Table 3 and shown in Fig. 3.

Ulysses was launched when the Earth was in the Meyer, Marsden \& Kracht region, which it left on January 1, 1991. Then it coasted outside the regions prone to detection of PUIs from the sungrazer population until Nov. 25, 1994, when it entered the Meyer region, which it exited on Dec. 13, 1994. Then it entered the Marsden \& Kracht region on Jan. 30, 1995 and the Kreutz region on Feb. 10, 1995, which it exited on May 14, 1995. This sequence was repeated during the second revolution of Ulysses around the Sun, entering the Meyer region on Feb. 02, 2001 and exiting it on Feb. 23, 2001, and entering the Marsden \& Kracht region on Apr. 12, 2001 and the Kreutz region on Apr. 23, 2001, where it stayed until Jul. 23, 2001. Ulysses dates and intervals are given in Table 4 and shown in Fig. 4.
If some of the inner source of pickup ions is indeed sungrazing comets, then in the time intervals indicated above one should expect seasonal changes of the inner source observed from $1 \mathrm{AU}$ both in the absolute flux and in the chemical composition. Ulysses should see the sungrazers' inner source on the intervals indicated in Fig. 4, which correspond well with the dates of PUI observations discussed by Gloeckler \& Geiss (1998, 2001) and Gloeckler et al. (2000a).

The size of the detection area of the Kreutz comets is about 2.39 sr, i.e. $\sim 0.19$ of the full solid angle. Hence the flux of all PUIs from the Kreutz comets averaged over time and the detection area should be $1.6 \times 10^{5} \mathrm{~g} \mathrm{~s}^{-1} \mathrm{sr}^{-1}$. Geiss et al. (1996) estimate the oxygen flux from the inner source as $2 \times 10^{6} \mathrm{~g} \mathrm{~s}^{-1}$ in the full solid angle, which gives $1.6 \times 10^{5} \mathrm{~g} \mathrm{~s}^{-1} \mathrm{sr}^{-1}-$ only two or three-fold higher given the mass fraction of oxygen in a cometary nucleus equal to $\sim 0.3$ (Greenberg \& Li 1999) and Gloeckler \& Geiss (1998) give $7 \times 10^{6} \mathrm{~g} \mathrm{~s}^{-1}$, i.e. $5.6 \times$ $10^{5} \mathrm{~g} \mathrm{~s}^{-1} \mathrm{sr}^{-1}$ - a higher value but still comparable to ours given the large uncertainty of all these estimates. 
Table 4. Detection intervals for sungrazing comet-related PUI and ENA for Ulysses ${ }^{a}$.

\begin{tabular}{lccccc}
\hline \hline $\begin{array}{l}\text { Comet group } \\
\text { event }\end{array}$ & $\begin{array}{c}\text { Meyer } \\
\text { in }\end{array}$ & $\begin{array}{c}\text { Meyer } \\
\text { out }\end{array}$ & $\begin{array}{c}\text { Marsden \& Kracht } \\
\text { in }\end{array}$ & $\begin{array}{c}\text { Kreutz } \\
\text { in }\end{array}$ & $\begin{array}{c}\text { Kreutz } \\
\text { out }\end{array}$ \\
\hline Date & Nov. 25 & Dec. 13, 1994 & Jan. 30, 1995 & Feb. 10, 1995 & May 14, 1995 \\
DOY & 329 & 347 & 30 & 41 & 134 \\
Ecliptic latitude $\left[{ }^{\circ}\right]$ & -72 & -60 & -33 & -26 & 41 \\
& & & & & \\
Date & Feb. 02 & 23,2001 & Apr. 12, 2001 & Apr. 23, 2001 & July 23, 2001 \\
DOY & 33 & 54 & 102 & 113 & 204 \\
Ecliptic latitude $\left[{ }^{\circ}\right]$ & -72 & -60 & -33 & -26 & 41 \\
\hline
\end{tabular}

${ }^{a}$ See also Fig. 4.

\subsection{Energetic neutral atoms}

One of the channels of PUI production from the sungrazers' material is charge exchange between solar wind protons and cometary neutrals. The reactions potentially important in the context of H ENA production are the following:

$\mathrm{H}+\mathrm{p} \rightarrow \mathrm{H}_{\mathrm{PUI}}^{+}+\mathrm{H}_{\mathrm{ENA}}$,

$\mathrm{O}+\mathrm{p} \rightarrow \mathrm{O}_{\mathrm{PUI}}^{+}+\mathrm{H}_{\mathrm{ENA}}$

and

$\mathrm{C}+\mathrm{p} \rightarrow \mathrm{C}_{\mathrm{PUI}}^{+}+\mathrm{H}_{\mathrm{ENA}}$,

where $\mathrm{H}, \mathrm{O}$, and $\mathrm{C}$ on the left-hand side are the cometary neutrals and $p$ are the solar wind protons. These reactions are practically the sole sources of Energetic Neutral Atoms of cometary origin since all other elements in the solar wind are highly ionized and cannot produce a neutral even if they exchange charge with a cometary neutral atom. We estimate the number $N_{\text {ENA }}$ of $\mathrm{H}$ ENAs produced by these reactions as a percentage of all atoms released by the breaking up nucleus and subsequently ionized and picked up by the solar wind as:

$N_{\mathrm{ENA}}=\sum_{A \in\{\mathrm{H}, \mathrm{O}, \mathrm{C}\}} \xi_{\mathrm{A}} X_{\mathrm{A}}$

where $X_{\mathrm{A}}$ is the number abundance of atoms A in the cometary nucleus and $\xi_{\mathrm{A}}$ is the percentage of the charge exchange rate $\beta_{\mathrm{A}, \mathrm{cx}}$ of element $\mathrm{A}$ to the total ionization rate of this element:

$\xi_{\mathrm{A}}=\beta_{\mathrm{A}, \mathrm{cx}} /\left(\beta_{\mathrm{A}, \mathrm{EUV}}+\beta_{\mathrm{A}, \mathrm{cx}}+\beta_{\mathrm{A}, \mathrm{el}}\right)$.

For the EUV rates we took mean values between solar minimum and maximum given by Ruciński et al. (1996), scaled to the appropriate distance according to $1 / r^{2}$. To compute the electron impact rates, we used the solar wind density and temperature model by Köhnlein (1996), with the proton density scaled up by $10 \%$ to account for electrons from the fullystripped elements heavier than hydrogen, and we assumed that the electron distribution function is mono-Maxwellian. For hydrogen, we calculated the electron impact rate following Ruciński \& Fahr (1989) and for oxygen and carbon following Phaneuf et al. (1987). To compute charge exchange, we used the aforementioned model of solar wind parameters by Köhnlein (1996) and velocity-dependent charge exchange cross sections between protons and $\mathrm{H}, \mathrm{O}$, and $\mathrm{C}$ atoms, respectively from Bzowski (2001), Stancil et al. (1999) and Stancil et al. (1998). We also computed the actual relative velocity between the typical Kreutz nucleus and the solar wind, which turned out to be weakly varying with the heliocentric distance and equal to $\sim 430 \mathrm{~km} \mathrm{~s}^{-1}$.

From the three ionization channels, we assembled the ionization budget of $\mathrm{H}, \mathrm{O}$, and $\mathrm{C}$ defined by Eqs. (7) and (8) at a few heliocentric distances between 1 and $0.01 \mathrm{AU}$. We found that percentage of the charge exchange rate in the net ionization rate of these ions in the solar neighbourhood depends only weakly on the heliocentric distance. For hydrogen, it increases from $\sim 66 \%$ at $0.1 \mathrm{AU}$ to $\sim 70 \%$ at $0.01 \mathrm{AU}$, for oxygen from $\sim 27 \%$ to $\sim 30 \%$ and for carbon from $\sim 7 \%$ to $\sim 10 \%$. Given the atomic abundances mentioned earlier, this yields the $\mathrm{H}$ ENA injection rate at the level of $\sim 40 \%$ of all PUIs injection rate in the nuclei breakup region.

Since in the charge exchange reactions in question there is no significant momentum exchange, the newly created H ENAs inherit the motion of the incident protons and escape. If this happens in the coma, the geometry is simple and the propagation close to radial (a "pencil beam"). Raymond et al. (1998) and Uzzo et al. (2001) point out a different possibility. They observed bright sungrazers close to and in the breakup region in the Lyman- $\alpha$ line using UVCS on SOHO. They observed a cometary linewidth of $\sim 200 \mathrm{~km} \mathrm{~s}^{-1}$, which they interpret not as caused by the $\mathrm{H}$ atoms emitted by the nucleus and travelling almost parallel to the comet towards the Sun, because those at these distances from the Sun are already Doppler-shifted outside the spectral range of the solar line, but as shocked solar wind protons, which were subsequently neutralized by chargeexchange with the new $\mathrm{H}, \mathrm{O}$, and $\mathrm{C}$ atoms from the nucleus. The velocity direction of these particles has been distributed widely by the cometary shock and some of them are inside the spectral range of the solar line. If such a scenario is realized, then a "shrapnel" geometry of propagation of these atoms is more appropriate, with atoms dispersed into the full antisolar hemisphere. The beam would be much widened at the expense of its flux magnitude. 
Solar wind accelerates in the breakup region from $\sim 50 \mathrm{~km} \mathrm{~s}^{-1}$ at $2.2 R_{\odot}$ to $\sim 400 \mathrm{~km} \mathrm{~s}^{-1}$ (Köhnlein 1996), so the $\mathrm{H}$ atoms starting off at $50 \mathrm{~km} \mathrm{~s}^{-1}$ at $2.2 R_{\odot}$ do not have enough energy to reach $1 \mathrm{AU}$ without support from the solar Lyman- $\alpha$ radiation pressure. The Doppler width of this self-reversed line is about $120 \mathrm{~km} \mathrm{~s}^{-1}$ (Lemaire et al. 2002) and the magnitude of the force exerted by solar photons exceeds the solar gravity force for radial velocities between $\sim 30 \mathrm{~km} \mathrm{~s}^{-1}$ and $\sim 75 \mathrm{~km} \mathrm{~s}^{-1}$. Hence, after very rapid acceleration from the start speed to the maximum speed at which the radiation pressure exceeds solar gravity, these atoms "surf" on the radiation pressure away from the Sun at a constant speed of $\sim 75 \mathrm{~km} \mathrm{~s}^{-1}(\sim 30 \mathrm{eV})$.

The atoms created farther away from the Sun have a larger initial speed because the seed solar wind protons are faster, and they are much less decelerated by solar gravity: those launched at $4 R_{\odot}$ have a speed of $120 \mathrm{~km} \mathrm{~s}^{-1}(75 \mathrm{eV})$ and immediately feel the solar radiation pressure, and those launched at $0.1 \mathrm{AU}$ have a speed of $320 \mathrm{~km} \mathrm{~s}^{-1}(0.53 \mathrm{keV})$ and at $1 \mathrm{AU}$ are slowed down to $\sim 290 \mathrm{~km} \mathrm{~s}^{-1}(0.44 \mathrm{keV})$, feeling no radiation pressure because they are Doppler-shifted away from the solar line.

Once created, the cometary H ENAs are subjected to ionization loss processes from all three hydrogen destruction channels. The EUV ionization and electron impact rates are similar to those of the original cometary $\mathrm{H}$ atoms. Charge exchange operates differently because of the different relative speeds between the local solar wind and the ballistic H ENAs. Since the speed of a newly-created H ENA is determined by the local solar wind speed and is further reduced by solar gravity while solar wind accelerates with the increase of heliocentric distance, the charge exchange rate is highest in the case of $\mathrm{H}$ ENAs created closest to the Sun. We estimated the survival probability of an H ENA injected at the typical breakup distance of $0.05 \mathrm{AU}$ and, for comparison, at $0.1 \mathrm{AU}$ (correspondingly, 10 and $20 R_{\odot}$ ). It turns out that practically all atoms launched at $0.05 \mathrm{AU}$ will be ionized (the survival probability will be $\sim 4 \times 10^{-4}$ ), while out of those launched at $0.1 \mathrm{AU} \sim 20 \%$ will be able to reach Earth's orbit.

Destruction of H ENAs created close to the Sun has another interesting consequence. When ionized, they will have a lower velocity than the surrounding solar wind and consequently they will be picked up. However, their injection velocity will not be 0 , so in the solar wind-related phase space they will be relatively close to the solar wind core, while retaining their pickup characteristics. Consequently, they will augment the original PUI population, created much closer to the Sun, and mimic PUIs created in the immediate solar neighborhood. Thus ENAs produced from charge exchange between solar wind protons and cometary neutrals will be another source of the inner source PUIs. Effectively, these will be protons extracted from the solar wind core and transported in phase space to the inner source PUI region.

The mean rate of $\mathrm{H}$ ENA production will be $8 \times$ $10^{26} \mathrm{H}$ atoms per second (40\% of the PUI production rate). Assuming the "shrapnel" geometry of initial propagation and considering the low probability of survival from the breakup region to $1 \mathrm{AU}$, we can expect a mean $\mathrm{H}$ ENA flux at Earth equal to just $10^{-4}$ at $\mathrm{s}^{-1} \mathrm{~cm}^{-2}$. In reality, the mean flux is not a good measure: we expect one comet every two days and the breakup takes about $2 \mathrm{~h}$. Therefore, actual instantaneous fluxes should be $\sim 25$-fold higher, i.e. $2.5 \times 10^{-3}$ at $\mathrm{s}^{-1} \mathrm{~cm}^{-2}$, however the durations should be just about $2 \mathrm{~h}$. For comparison, the H ENA flux from interstellar hydrogen at the offset angle $\sim 120^{\circ}$ from the upwind direction is expected to be of the order of 1 to 50 at $\mathrm{s}^{-1} \mathrm{~cm}^{-2}$, depending on the phase of solar cycle (Bzowski et al. 1996). Hence, the H ENAs from comets would be observable at Earth only close to solar maximum, and with luck. However, they will be a source of the extra $25 \%$ of the sungrazer PUI flux on top of the PUI originating from the cometary material itself.

\section{Discussion}

The suggestion that comets might be responsible for the inner source of pickup ions was proposed quite early (Geiss et al. 1995), although the primary mechanism envisaged was loss of volatile components at larger distances from the Sun. Frequently appearing small comets from isotropic directions would be hardly distinguishable from an extended source of particles (Gloeckler \& Geiss 1998).

The composition of the inner source shows depletion of hydrogen with respect to solar wind abundances. Schwadron \& Geiss (2000) extensively discuss possible mechanisms of this depletion under the assumption that most of the inner source hydrogen is released from dust grains as $\mathrm{H}_{2}$ molecules. If, however, we take the sungrazing comets as the inner source, the problem of hydrogen underabundance is strongly alleviated, even with the extra PUIs from the reionized cometary H ENAs taken into account.

The chemical composition of the inner source PUI inferred by Gloeckler \& Geiss (1998) and Gloeckler et al. (2000a) and discussed extensively by Schwadron et al. (2000) shows, among others, helium and neon. Noble gases are not expected in "old" dust grains near the Sun and hence a mechanism of absorption of solar wind neon ions to the dust grains and its further release after recombination was proposed. Sungrazers do not alleviate this problem directly: very little, if any at all of atoms of noble gases were found among cometary volatiles (Geiss et al. 1999; Boclée-Morvan et al. 2004). Since, however, they supply dust to the immediate solar neighbourhood then the same mechanism that neutralizes solar wind neon on solar system dust grains should operate on the dust grains from comets.

The evolution of PUIs from the inner source both with respect to the phase space and to the charge state was discussed by Schwadron et al. (2000). They point out that during the drift away from the Sun the PUI should experience more or less adiabatic cooling which would make it increasingly difficult to observe them with the increasing distance to the Sun. Therefore, in the case of Ulysses, the best time seems to be when the spacecraft is close to its perihelion, i.e., in the portion of the orbit close to the "fast latitude scan".

In the estimates of the PUI flux we took an average production rate. In reality, the sungrazer PUI flux will be highly fluctuating both in time and in space. On the one hand, one can expect that even when a comet is as close to the Sun as $0.2 \mathrm{AU}$, the source region for PUIs should be large enough to result in a few days' duration of the PUI signal at the Earth 
or Ulysses orbit (Gloeckler et al. 2004). Indeed, Gloeckler et al. (2000b) observed with Ulysses the PUI tail of Comet C/1996 B2 Hyakutake when it was at $\sim 0.35$ AU from the Sun and the signal (apart from a $\sim 1$ day peak) seems to last for 5-6 days. On the other hand, the average apparition rate for the Kreutz group is one every other day (and of the secondary groups one per month) and the apparition times show a Poisson distribution. Therefore it cannot be ruled out that the inner source of PUIs from the Kreutz sungrazers will be intermittently drying out and that the PUIs from the secondary groups will not be detected at all.

Further, we assumed a radial propagation of the populations created from the cometary material, which need not be true. While PUIs from large sungrazers can probably be observed as well-defined signals (Iseli et al. 2002), the fluxes from the much more frequent smaller objects can be so low that they will be registered as singular events, hard to notice on a case by case basis and showing up only when a comparison of long-time averages is made. A good test of the reality of the cometary character of the inner source would be to average available PUI and possibly ENA observations over the time intervals proposed in this paper and to compare the results with an averaging performed outside these time frames.

\section{Conclusions}

We suggest that a considerable portion of the inner source of pickup ions may originate from the SOHO sungrazing comets, especially from the Kreutz group. We show that the actual rate of inflow of these comets may be as high as one per two days. The corresponding total mass production rate would be $3.1 \times 10^{4} \mathrm{~g} \mathrm{~s}^{-1}$, released into the solar wind between $\sim 40 R_{\odot}$ and $\sim 2 R_{\odot}$ and emitted into a restricted area of $\sim 0.19$ of the full volume angle, so that the flux is $1.6 \times 10^{5} \mathrm{~g} \mathrm{~s}^{-1} \mathrm{sr}^{-1}$. Together with the re-ionized $\mathrm{H}$ ENAs created by charge exchange between cometary atoms and solar wind protons, this gives a mean PUI particle flux equal to $1.2 \times 10^{27} \mathrm{~s}^{-1} \mathrm{sr}^{-1}$, which at $1 \mathrm{AU}$ is equivalent to $12.5 \mathrm{~s}^{-1} \mathrm{~cm}^{-2}$. The intensity of sungrazerrelated populations should be strongly variable both in time and in space. Because of the geometry of the orbits, the pickup ions should be observable by an Earth- or L1-orbiting spacecraft between end of July and the end of the year. Ulysses should have been within the detection area since its launch until the end of 1990 (but because of the scarcity of this source it could have missed them), and then during its two fast latitude scans between $\sim-25^{\circ}$ and $\sim 40^{\circ}$ ecliptic latitude, with a plunge into a low detection-probability area between $\sim-75^{\circ}$ and $\sim-60^{\circ}$ latitude. Measurements of the inner source pickups carried out within these areas should show different intensities and characteristics than when performed elsewhere. To our knowledge, the cometary hypothesis is the only one that predicts a distinct ecliptic longitude effect and as such should be easily verifiable by inspection of existing PUI observations from Ulysses and 1 AU spacecraft, as Wind and ACE.

Acknowledgements. The authors gratefully acknowledge discussions with Grzegorz Sitarski, Andrzej Czechowski, and Stan Grzedzielski from the Space Research Centre PAS, and Eberhard Möbius from the
University of New Hampshire. This research was supported by the Polish State Committee for Scientific Research Grant 1 P03D 00927.

\section{References}

Biesecker, D. A., Lamy, P., Cyr, O. C. S., Llebaria, A., \& Howard, R. A. 2002, Icarus, 157, 323

Boclée-Morvan, D., Crovisier, J., Mumma, M. J., \& Weaver, H. A. 2004, The composition of cometary volatiles, ed. M. C. Festou, H. U. Keller, \& H. A. Weaver (University of Arizxona Press), 1

Budzien, S. A., Festou, M. C., \& Feldman, P. D. 1994, Icarus, 107, 164

Bzowski, M. 2001, Space Sci. Rev., 97, 379

Bzowski, M., Fahr, H. J., \& Ruciński, D. 1996, Icarus, 124, 209

Fahr, H. J. 1973, Sol. Phys., 30, 193

Geiss, J., \& Altweg, K. 1998, ESA SP, 431, 103

Geiss, J., Gloeckler, G., Mall, U., et al. 1994, A\&A, 282, 924

Geiss, J., Gloeckler, G., Fisk, L. A., \& von Steiger, R. 1995, J. Geophys. Res., 100, 23373

Geiss, J., Gloeckler, G., \& von Steiger, R. 1996, Space Sci. Rev., 78, 43

Geiss, J., Altwegg, K., Balsiger, H., \& Graf, S. 1999, Space Sci. Rev., 90, 253

Gloeckler, G., \& Geiss, J. 1998, Space Sci. Rev., 86, 127

Gloeckler, G., \& Geiss, J. 2001, Space Sci. Rev., 97, 169

Gloeckler, G., Hovestadt, D., Ipavich, F. M., et al. 1986, Geophys. Res. Lett., 13, 251

Gloeckler, G., Geiss, J., Balsiger, H., et al. 1993, Science, 261, 70

Gloeckler, G., Fisk, L. A., Geiss, J., Schwadron, N. A., \& Zurbuchen, T. H. 2000a, J. Geophys. Res., 105, 7459

Gloeckler, G., Geiss, J., Schwadron, N. A., et al. 2000b, Nature, 404, 576

Gloeckler, G., Allegrini, F., Elliott, H. A., et al. 2004, ApJ, 604, L121

Greenberg, J. M., \& Li, A. 1999, Space Sci. Rev., 90, 149

Grünwaldt, H., Neugebauer, M., Hilchenbach, M., et al. 1997, Geophys. Res. Lett., 24, 1163

Huebner, W. F., Keady, J. J., \& Lyon, S. P. 1992, Ap\&SS, 195, 1

Iseli, M., Küppers, M., Benz, W., \& Bochsler, P. 2002, Icarus, 155, 350

Kimura, H., Mann, I., Biesecker, D. A., \& Jessberger, E. K. 2002, Icarus, 159,529

Köhnlein, W. 1996, Sol. Phys., 169, 209

Kreutz, H. 1888, Publ. Sternwarte Kiel, 3, 1

Kreutz, H. 1891, Publ. Sternwarte Kiel, 6, 1

Kreutz, H. 1901, Astron. Abhand., 1, 1

Lemaire, P. L., Emerich, C., Vial, J. C., et al. 2002, in From Solar Min to Max: Half a Solar Cycle with SOHO, ESA SP-508, 219

Luhmann, J. G. 2003, ApJ, 592, 1241

Luhmann, J. G., Fedder, J. A., \& Winske, D. 1988, J. Geophys. Res., 93, 7532

Mäkinen, J. T. T., Bertaux, J., Combi, M. R., \& Quémerais, E. 2001a, Science, 292, 1326

Mäkinen, J. T. T., Bertaux, J.-L., Pulkkinen, T. I., et al. 2001b, A\&A, 368,292

MacQueen, R. M., \& St. Cyr, O. C. 1991, Icarus, 90, 96

Mann, I., Kimura, H., Biesecker, D. A., et al. 2004, Space Sci. Rev., 110,269

Marsden, B. 1967, AJ, 72, 1170

Marsden, B. 1989, AJ, 98, 2306

Marsden, B., \& Williams, G. 2003, Catalogue of Cometary Orbits, XVth edition, ed. G. Brian, Marsden, Gareth, \& V. Williams

Michels, D. J., Sheeley, N. R., Howard, R. A., \& Koomen, M. J. 1982, Science, 215, 1097 
Möbius, E., Hovestadt, D., Klecker, B., et al. 1985, Nature, 318, 426

Phaneuf, R. A., Janev, R. K., \& Pindzola, M. S. 1987, Atomic data for fusion, Vol. ORNL-6086/V5 (Oak Ridge, Tenn.: Oak Ridge National Laboratories)

Povich, M. S., Raymond, J. C., Jones, G. H., et al. 2003, Science, 302, 1949

Raymond, J. C., Fineschi, S., Smith, P. L., et al. 1998, ApJ, 508, 410

Ruciński, D., Cummings, A. C., Gloeckler, G., et al. 1996, Space Sci. Rev., 78, 73

Ruciński, D., \& Fahr, H. J. 1989, A\&A, 224, 290

Schwadron, N. A., \& Geiss, J. 2000, J. Geophys. Res., 105, 7473
Schwadron, N. A., Geiss, J., Fisk, L. A., et al. 2000, J. Geophys. Res., 105,7465

Sekanina, Z. 2002, ApJ, 566, 577

Sekanina, Z. 2003, ApJ, 597, 1237

Sheeley, N. R., Howard, R. A., Koomen, M. J., \& Michels, D. J. 1982, Nature, 300, 239

Stancil, P. C., Havener, C. C., Krstić, P. S., et al. 1998, ApJ, 502, 1006

Stancil, P. C., Schultz, D. R., Kimura, M., et al. 1999, A\&AS, 140, 225

Uzzo, M., Raymond, J. C., Biesecker, D., et al. 2001, ApJ, 558, 403 